\title{
Risk factors associated with multidrug resistant tuberculosis among patients referred to Kibong'oto Infectious Disease Hospital in northern Tanzania
}

\author{
NSIANDE A. LEMA ${ }^{1}$, MTEBE MAJIGO ${ }^{2 *}$, PETER M. MBELELE³, AHMED ABADE ${ }^{1} \&$ MECKY I. MATEE ${ }^{2}$ \\ ${ }^{1}$ Field Epidemiology and Laboratory Training Programme, Dar es Salaam, Tanzania \\ ${ }^{2}$ Department of Microbiology and Immunology, Muhimbili University of Health and Allied Sciences, P.O. Box \\ 65001, Dar es Salaam, Tanzania \\ ${ }^{3}$ Kibong'oto Infectious Disease Hospital, P.O. Box 12, Sanya Juu, Tanzania
}

\begin{abstract}
Background: Multidrug resistant tuberculosis (MDR-TB) has remained an important public health problem in developing world. We conducted this study to determine risk factors associated with MDR-TB and drug susceptibility pattern to second line drug among MDR TB patients in Tanzania.

Methods: From July to November 2014, Unmatched case control study was conducted at Kibong'oto Infectious Diseases Hospital in Tanzania. A case was defined as any patient whose sputum yielded Mycobacterium tuberculosis that were resistance to at least rifampin (RFP) and isoniazid (INH) whereas control was defined as those sensitive to rifampin (RFP) + isoniazid (INH). One morning sputum sample was collected from each study participant and cultured on Löwenstein-Jensen (LJ) media for $M$. tuberculosis. Drug susceptibility testing of isolated $M$. tuberculosis was done for rifampicin, isoniazid, kanamycin and ofloxacin. A semi-structured questionnaire was used to collect socio-demographic and risk factors information for MDR-TB.

Results: A total of 102 cases and 102 controls were enrolled. The predominant age group was 31- 40 years, of whom cases and controls accounted for 38 (37.3\%) and 35 (34.3\%) of study participants, respectively. Majority of participants ( $69 \%$ cases and $71 \%$ control) were males and self-employed $(62.7 \%$ cases and $84.4 \%$ controls). More than half (52\%) and approximately a quarter (24.5\%) of cases and control had HIV infection, respectively. About two-thirds of cases (62.7\%) were cigarette smokers where by cigarette smoking was reported in $42.2 \%$ of controls. Previous history of TB treatment accounted for approximately three quarter (72.5\%) and only $24.5 \%$ of cases and controls, respectively. Risk factors independently associated with MDRTB were previous history of treatment with first line anti-TB $(O R=3.3,95 \% \mathrm{Cl} 1.7-6.3)$, smoking $(\mathrm{OR}=1.9,95 \%$ $\mathrm{Cl}$ 1.0-3.5), contact with TB case (OR=2.7, 95\% $\mathrm{Cl}$ 1.4-5.1) and history of TB. All MDR TB isolates were sensitive to kanamycin and ofloxacin.

Conclusion: MDR-TB among patients referred to Kibong'oto Infectious Diseases Hospital is associated with previous history of TB contact, smoking habit, and contact with TB case. All MDR TB isolates were sensitive to the tested second line drugs, Kanamycin and Ofloxacin.
\end{abstract}

Keywords: Tuberculosis, MDR-TB, risk factors, drug resistance, second line anti-TB, Tanzania

\section{Introduction}

Tuberculosis (TB), an inhalational infectious disease caused by Mycobacterium tuberculosis is still a global health problem (WHO, 2015). Its control has been jeopardized by emergence of drug resistant (DR) M. tuberculosis strains and Human Immunodeficiency Virus (HIV) infection with or without Acquired Immunodeficiency Syndrome (AIDS) (Andrews et al., 2010). Multi-Drug resistance TB (MDR-TB), defined as resistance to at least rifampicin and isoniazid the two key first line drugs used to treat drug susceptible TB in combination with other first line drugs (pyrazinamide, ethambutol, streptomycin) has emerged as a global threat (WHO, 2014, 2015) and thus, calling for use of second line drugs (SLD) that include kanamycin, capromycin, levofloxacin, cycloserine and ethionamide. However, these drugs are toxic and yet less effective and hence, encouraging prolonged treatment duration for a minimum of 20 months (WHO, 2014). Emergence of $M$. tuberculosis resistant strains to anti-TB drugs may develop either through spontaneous chromosomal mutation in specific gene targets or following inappropriate use of anti-TB drugs, resulting to selection pressure and inadequate drug exposure (WHO, 2014). 
Globally, the prevalence of MDR TB in new and previously treated TB cases is $3.5 \%$ and 20.5\%, respectively, with Sub-Saharan Africa (SSA) contributing the highest proportion (WHO, 2015). In Tanzania, the National TB drug surveillance of 2006 found that, the prevalence of MDR TB in new and previously treated cases were $1.1 \%$ and 3.9\%, respectively (Chonde et al., 2010). MDR-TB has been described as a man-made problem resulting from the use of inadequate drug regimen that selects drug resistant tubercle bacilli, with several factors being linked to propagate its emergence (Andrew et al., 2010; El Mahalli \& Al-Qahtani, 2015; WHO, 2014, 2015). For example, previous history of TB treatment has been documented as an important risk factor that predicts emergence of MDR-TB (Andrews et al., 2010). In addition, poor patient adherent to anti-TB treatment (WHO, 2006), HIV infection, young age, life style such as smoking, illicit drug use and imprisonment (Andrew et al., 2010; El Mahalli \& AL-Qahtani, 2015) have also been reported to predict emergence of MDR-TB. Nevertheless, these factors have been inadequately studied in Tanzania.

Drug resistance TB (DR-TB such as MDR-TB, XDR-TB) is a laboratory diagnosis that is confirmed either by first isolating $M$. tuberculosis on conventional culture followed by drug susceptibility testing (WHO, 2015) or molecular diagnostic test such as GenoTypeMTBDRplus (HainLifescience GmbH, Nehren, Germany) or Cepheid GeneXpert MTB/RIF (Cepheid, Sunnyvale, CA) that detects mutation in specific $M$. tuberculosis gene targets conferring resistance to anti-TB drugs (first or second line drugs) (Bohme et al., 2011; Ao et al., 2012; Steingart et al., 2014). Most laboratories in resource limited countries like Tanzania have access to drug susceptibility testing (DST) for first line drugs (Streptomycin, Isoniazid, Rifampicin and Ethambutol abbreviated as SIRE) only. In Tanzania, despite the paucity information regarding second line drug susceptibility profile, patients confirmed to harbour $M$. tuberculosis resistant strains to Rifampicin with or without Isoniazid (MDR TB), are empirically treated using a standardized second line regimen (WHO, 2013). This subjects patients to toxic and yet less effective drugs for prolonged period of time as a result, poor adherence to treatment ensue and hence, creating good environment for emergence of more severe form of DR TB like XDR-TB (WHO, 2014). We hypothesized that understanding risk factors for developing MDR-TB and treating with appropriate anti-TB drugs is critical in designing intervention strategies that are best suited towards TB control in Tanzania. Therefore, this study was conducted to determine risk factors associated with MDR-TB and drug susceptibility pattern to second line drug among patients with MDR TB in Tanzania.

\section{Materials and Methods}

\section{Study area and design}

This unmatched case control study design was conducted from July to November 2014 at Kibong'oto Infectious Diseases Hospital (KIDH) in northern Tanzania. KIDH is a centre of excellence for MDR TB management in the country, with bed capacity of 340 and receives all MDR-TB patients for care and treatment from all parts of Tanzania.

\section{Study population and sampling method}

A total of 102 cases defined as patients with MDR-TB (resistant to at least Rifampicin and isoniazid) and 102 controls defined as patients with susceptible TB (having M. tuberculosis that is susceptible to all first line drugs, SIRE) confirmed either by conventional culture and DST or molecular test such as GeneXpert MTB/Rif assay, Line Probe Assay (MTBDRplus HainLifescicnce) using existing protocols, were recruited to participate in the study. Before enrolment, cases and controls were obtained using simple random sampling method from a hospital list. Different numbers were assigned followed by generating random numbers on Open Epi Random Program for cases and controls. Random numbers corresponding to either case or control were picked by the open Epi and enrolled in the study. 


\section{Data collection}

Information regarding socio-demographic characteristics such as age, sex, and marital status, and clinical information such as previous history of TB treatment, HIV status and risk factors including smoking, use of illicit drug, history of being prisoned, and alcohol intake was obtained using a pretested standardized structured questionnaire.

\section{Laboratory Investigation}

At least $3-5 \mathrm{ml}$ of one morning quality sputum sample was collected in a $50 \mathrm{ml}$ falcon tube from all participants. Quality sputum was indicated by the presence of mucoid or mucopulurent material, sufficient volume and absence of either saliva or food particles. Participant's particulars were verified before sputum sample was delivered to the Laboratory. Sputum sample was stored at 2$8^{\circ} \mathrm{C}$ for 24 hours before properly packed and transported under stable temperature condition to Central Referral Tuberculosis Laboratory (CRTL) for culture and Drug susceptibility testing (DST) using standards protocol as provided by CRTL. At CTRL, fresh digestant was prepared by adding equal amounts of $4 \% \mathrm{NaOH}$ and $2.9 \%$ sodium citrate, followed by $0.5 \mathrm{~g} \mathrm{NALC}$ powder per $100 \mathrm{~mL}$ of sodium hydroxide-sodium citrate solution. The $\mathrm{NaOH}-\mathrm{NALC}$ solution was then added in equal volume to sputum sample collected in a $50 \mathrm{~mL}$ plastic centrifuge tube, mixed well and left to stand for 20 minutes. Phosphate buffer at $\mathrm{pH} 6.8$ was then added up to $50 \mathrm{ml}$ mark and the sample was concentrated by centrifugation at $3000 \mathrm{~g}$ for 15 minutes. The supernatant was discarded and deposits were inoculated on both pyruvate and glycerol containing LöwensteinJensen ( $L J)$ media slants. In parallel, a standard laboratory strain, M. tuberculosis (ATCC H37Rv) and un-inoculated $\mathrm{L}$ media for sterility and for growth as positive and negative control. All $L$ slants were incubated at $37^{\circ} \mathrm{C}$ for up to 8 weeks before declared culture negative. All positive culture slopes were assessed for $M$. tuberculosis growth rate, colony morphology, pigment production and smear microscopy for acid fast bacilli.

Susceptibility testing for kanamycin and ofloxacin was performed on $M$. tuberculosis isolates that shown resistance to isoniazid and rifampicin (MDR-TB cases) using predefined standard operating procedure for proportion method at CTRL. An isolate was considered sensitive if there was no growth or presence of less than $1 \%$ of colonies growing as compared to the drug free controls isolate. Details of the culture and DST procedures as well as quality considerations have been previously described by Matee et al. (2008).

\section{Data analysis}

Data were coded, entered, cleaned and analysed using Epi-Info version 3.5 Statistical software. Descriptive analysis was done by running frequencies, creating 10-interval age groups and quartiles of study variables. The respondents were categorized into cases (MDR-TB) and controls (susceptible TB). Odds ratio was used to measure the strength of association between outcome and exposure (risk factors) at $95 \%$ confidence interval. Risk factor variables with $p<0.05$ was considered as having significant association with MDR-TB. All factors with $p \leq 0.2$ at bivariate analysis were entered into unconditional logistic regression model to generate independent factors associated with development of MDR-TB.

\section{Ethical consideration}

Approval and clearance was obtained from Muhimbili University of Health and Allied Sciences Research and Publications Committee. All study participants gave a written informed consent before they were enrolled into the study. Information obtained was treated with confidentiality that only researchers had access to filled questionnaire and electronic data. All Patients with MDR-TB (cases) were treated according to existing National MDR-TB treatment guidelines at $\mathrm{KIDH}$. 


\section{Results}

Socio demographic and clinical characteristics of study participants

A total of 102 cases and 102 controls were recruited, with males accounting for over two third of either cases or controls. Majority of study participants were in the age group of 31- 40 years, of whom 38 (37.3\%) and 35(34.3\%) were cases and controls, respectively. More than half (53; 52\%) and approximately a quarter $(25 ; 24.5 \%)$ of cases and control had HIV infection, respectively. About two-thirds $(62.7 \%)$ of cases and $42.2 \%$ of controls were cigarette smokers. Previous history of TB treatment accounted for $72.5 \%$ of cases (Table 1 ).

Table 1: Socio-demographic characteristics and clinical information of cases and controls

\begin{tabular}{|c|c|c|c|c|c|}
\hline \multirow[t]{2}{*}{ Characteristics } & \multirow[t]{2}{*}{ Response } & \multicolumn{3}{|c|}{ Case $(\mathrm{N}=102)$} & \multirow{2}{*}{$\begin{array}{l}\text { Control }(\mathrm{N}=102) \\
\text { Percent }\end{array}$} \\
\hline & & Number & Percent & Number & \\
\hline \multirow[t]{2}{*}{ Sex } & Female & 31 & 30.4 & 30 & 29.4 \\
\hline & Male & 71 & 69.6 & 72 & 70.6 \\
\hline \multirow[t]{6}{*}{ Age group (years) } & $0-20$ & 10 & 9.8 & 8 & 7.9 \\
\hline & $21-30$ & 17 & 16.7 & 14 & 13.7 \\
\hline & $31-40$ & 38 & $37 \cdot 3$ & 35 & $34 \cdot 3$ \\
\hline & $41-50$ & 20 & 19.6 & 28 & 27.5 \\
\hline & $51-60$ & 10 & 9.8 & 11 & 10.8 \\
\hline & $>60$ & 7 & 6.9 & 6 & 5.9 \\
\hline \multirow[t]{4}{*}{ Education } & None & 16 & $15 \cdot 7$ & 19 & 18.6 \\
\hline & Primary & 57 & 55.9 & 72 & 70.6 \\
\hline & Secondary & 25 & 24.5 & 11 & 10.8 \\
\hline & Tertiary & 4 & 3.9 & 0 & 0 \\
\hline \multirow[t]{6}{*}{ Marital status } & Cohabiting & 3 & 2.9 & 4 & 3.9 \\
\hline & Divorce & 7 & 6.9 & 3 & 2.9 \\
\hline & Married & 45 & 44.1 & 53 & 52.0 \\
\hline & Separated & 6 & 5.9 & 9 & 8.8 \\
\hline & Single & 37 & 36.3 & 28 & 27.5 \\
\hline & Widowed & 4 & $3 \cdot 9$ & 3 & 2.9 \\
\hline \multirow[t]{4}{*}{ Employment status } & Employed & 20 & 19.6 & 10 & 9.8 \\
\hline & Not employed & 12 & 11.8 & 5 & 4.9 \\
\hline & Self employed & 64 & 62.7 & 83 & 81.4 \\
\hline & Students & 5 & 4.9 & 4 & 3.9 \\
\hline \multirow[t]{2}{*}{ HIV status } & Positive & 53 & 52.0 & 25 & 24.5 \\
\hline & negative & 49 & 48.0 & 77 & 75.5 \\
\hline \multirow[t]{2}{*}{ History of smoking } & Yes & 64 & 62.7 & 43 & 42.2 \\
\hline & No & 38 & $37 \cdot 3$ & 59 & 57.8 \\
\hline \multirow[t]{2}{*}{ Contact with TB case } & Yes & 53 & 52.0 & 25 & 24.5 \\
\hline & No & 49 & 48.0 & 77 & $75 \cdot 5$ \\
\hline \multirow[t]{2}{*}{ History of TB treatment } & Yes & 74 & 72.5 & 25 & 24.5 \\
\hline & No & 28 & 27.5 & 77 & $75 \cdot 5$ \\
\hline
\end{tabular}

Risk factors associated with MDR-TB and second line drug susceptibility testing

Crude Odds ratios showed that patients with previous history of treatment with first line anti-TB drugs (OR=8.1, $95 \% \mathrm{Cl}$ 4.3-15.2), smoking ( $\mathrm{OR}=2.3,95 \% \mathrm{Cl} 1.2-4.2)$, larger family size ( $>5$ members) (OR=2.1, 95\% Cl 1.1), HIV infection ( $\mathrm{OR}=8.1,95 \%, \mathrm{Cl} 1.8-36.7)$ and imprisonment (OR=4.4, 95\%, $\mathrm{Cl}$ 2.19.3) were risk factors associated with developing MDR TB (Table 2). When confounders were controlled, previous history of treatment with first line anti-TB drugs (OR=3.3, 95\% Cl 1.7-6.3), smoking (OR=1.9, $95 \% \mathrm{Cl}$ 1.0-3.5), contact with TB case (OR=2.7, 95\% $\mathrm{Cl} 1.4-5.1)$ and history of TB treatment were the independent factors associated with MDR TB (Table 2). All MDR TB isolates were sensitive to second line drugs (kanamycin and ofloxacin). 
Table 2: Risk factors associated with MDR-TB

\begin{tabular}{|c|c|c|c|c|c|}
\hline Variable & Response & $\begin{array}{l}\text { Cases } \mathrm{N}=102 \\
\mathrm{n}(\%)\end{array}$ & $\begin{array}{l}\text { Controls } \mathrm{N}=102 \\
\mathrm{n}(\%)\end{array}$ & $\mathrm{COR}^{*}(95 \% \mathrm{Cl})$ & $\mathrm{aOR}^{2}(95 \% \mathrm{Cl})$ \\
\hline \multirow[t]{2}{*}{ Ever smoked } & Yes & $64(52.0)$ & $43(53.1)$ & 1 & 1 \\
\hline & No & $38(46.9)$ & $59(48.0)$ & $2.3(1.2-4.2)$ & $1.9(1.0-3.5)$ \\
\hline \multirow[t]{2}{*}{ History of imprisonment } & Yes & $43(42 \%)$ & $14(13 \%)$ & 1 & 1 \\
\hline & No & $59(57.8 \%)$ & $85(83 \%)$ & $4 \cdot 4(2.1-9 \cdot 3)$ & $1.5(0.7-3.4)$ \\
\hline \multirow[t]{2}{*}{ Take alcohol } & Yes & $46(46.0)$ & $54(54.0)$ & 1 & \\
\hline & No & $56(53.8)$ & $48(46.2)$ & $0.7(0.4-1.33)$ & \\
\hline \multirow[t]{2}{*}{ Drug addicted } & Yes & $9(40.9)$ & $13(59.1)$ & 1 & \\
\hline & No & $93(51.7)$ & $87(48.3)$ & $0.6(0.2-1.5)$ & \\
\hline \multirow[t]{2}{*}{ Contact with TB case } & Yes & 53 & 25 & 1 & 1 \\
\hline & No & 49 & 77 & $3.3(1.7-6.3)$ & $2.7(1.4-5.1)$ \\
\hline Positive & Positive & 97 & 89 & 1 & 1 \\
\hline Negative & Negative & 2 & 15 & $8.1(1.8-36.7)$ & $1.7(0.9-1.2)$ \\
\hline $\begin{array}{l}\text { Ever being in refugee } \\
\text { camp }\end{array}$ & Yes & $7(53.8)$ & $6(46.2)$ & 1 & 1 \\
\hline No & No & $95(50.8)$ & $92(49.2)$ & $1.12(0.3-3.4)$ & $0.3(0.08-1.3)$ \\
\hline \multirow[t]{2}{*}{ TB disease type } & Pulmonary & $100(51)$ & $94(48)$ & 1 & 1 \\
\hline & Extra-pulmonary & $2(20)$ & $8(80)$ & $4.2(0.8-29.8)$ & $6.5(1.2-35.2)$ \\
\hline \multirow[t]{2}{*}{ Size of family $>5$} & Yes & $50(49.0)$ & $32(31.3)$ & & \\
\hline & No & $52(50.9)$ & $70(68.6)$ & $2.1(1.1-3.8)$ & $1.7(0.9-1.2)$ \\
\hline \multirow[t]{2}{*}{ History of treatment } & Yes & $74(74.7)$ & $25(25 \cdot 3)$ & & \\
\hline & No & $28(26.7)$ & $77(73 \cdot 3)$ & $8.1(4 \cdot 3-15 \cdot 2)$ & \\
\hline
\end{tabular}

Key: *cOR, odds ratio, ^aOR, adjusted odds ratio, $\mathrm{Cl}$, confidence interval

\section{Discussion}

In this study a number of factors were significantly associated with the occurrence of MDR-TB. We found that, history of previous TB treatment was the strongest independent risk factor associated with MDR-TB, which is in line with findings from other studies (Barroso et al., 2003; Zignol et al., 2006; Marahatta et al., 2010; Chiang et al., 2007; Andrews et al., 2010; El Mahalli \& ALQahtani, 2015). This imply that the usual practice of re-treating TB patient with first-line anti-TB drugs without DST results is not an effective approach and may contribute to the persistence and continued spread MDR-TB strains in the community. This hypothesis is supported by the fact that MDR-TB cases were 3.3 more likely to have previous contact history with a TB patient than controls. Similar findings have been reported elsewhere (Barroso et al., 2003). We also found that the odds of developing MDR-TB among smokers was 2.3 times higher compared with nonsmokers, which is also in line with the findings of others studies that showed cigarette smoking to have an increased risk of developing MDR TB (Barroso et al., 2003; Chiang et al., 2007; Marahatta et al., 2010). Having a larger size of family was associated with an increased likelihood of developing MDR-TB, indicating possibility of intra-familial transmission of resistant strains. Previous studies have reported that overcrowding, poor housing, and poor ventilation are factors that supports transmission of tuberculosis, including MDR-TB (Marahatta et al., 2010; Liang et al., 2012).

In the current study being HIV infection was not independently associated with MDR-TB, similar to a previous study conducted in Tanzania (NTLP, 2013) and in other parts of the world (Churchyard et al., 2000; Barroso et al., 2003; Marahatta et al., 2010; Andrews et al., 2010; Liang et al., 2012; El Mahalli \& AL-Qahtani, 2015). This finding is however deviates from other studies that have found association between HIV infection and MDR-TB (Andrews et al. 2010). The difference 
is likely to have been attributed to variation in HIV prevalence between our study and other studies. In addition, a study by Andrews et al. (2010) was conducted in population with high HIV and MDR-TB prevalence as well. Already, some studies have shown that drug-resistant TB risk factors are likely to differ in settings with low and high prevalence of HIV and MDR-TB (Escombe et al., 2008; Dean et al. 2014). So whether HIV infection per se increases the risk of drug resistance remains unclear, especially during this era of universal access to antiretroviral therapy and prophylaxis to infectious diseases.

Interestingly, none of the MDR-TB isolates was resistant to second line drugs tested (kanamycin and ofloxacin). This finding is in agreement to the fact that, since 2009 when Tanzania started implementing MDR TB management, there has been only one extensive drugresistant TB (XDR TB) case reported in the country (R.M. Kisonga, per comm), and even the most recent study has shown low levels of MDR and XDR TB among TB patients in Tanzania (Nagu et al., 2015). However, the government of Tanzania should continuously be vigilant in surveillance for possible emergence of XDR TB strains.

This study has some limitations which include the fact that CD4 counts were not performed for the HIV-infected individuals to determine extent of immune suppression and that the risk factors and drug-resistance were measured at the same time, which may not provide a temporal association.

Using these findings, we can conclude that, previous history of TB treatment, smoking habit and history of contact with TB case were the risk factors associated with MDR-TB in patients referred to Kibong'oto Infectious Diseases Hospital. Therefore, these factors particularly smoking, which is rarely emphasized in TB control strategies should be considered when formulating TB control policy to monitor patients with drug resistance TB. In addition, further studies should investigate mechanisms of how these predictors affect drug resistance.

\section{Competing interests}

The authors declare that they have no competing interests.

\section{Authors' contributions}

NAL: conceived the study, designed the study, collected data, statistical analysis, manuscript writing. MM: designed the study, data interpretation, reviewed and wrote the manuscript. PMM: Prepared the first draft, made interpretation of data, reviewed and compiled the final version of manuscript. AA: designed the study, performed statistical analysis and contributed in manuscript writing. MIM: supervised the whole process from study design, execution to manuscript writing. All authors read, approved the final version of manuscript and agreed to be accountable for all aspects of the work published.

\section{Acknowledgements}

The authors would like to thank the Director of Kibong'oto Infectious Diseases Hospital, Dr. Riziki M. Kisonga for giving permission to conduct this study, laboratory manager CTRL, Basra Doulla and Geofrey Mwenda for their support during the laboratory work. We also thank the Ministry of Health, Community Development, Gender, Elderly and Children for financial support.

\section{References}

Andrews, J.R., Shah, N.S., Weissman, D., Moll, A.P., Friedland, G. \& Gandhi, N.R. (2010) Predictors of multidrug and extensively drug-resistant tuberculosis in a high HIV prevalence community. PLoS One 5(12): 1-6 
Ao, W., Aldous, S., Woodruff, E., Hicke, B., Rea, L., Kreiswirth, B. \& Jenison, R. (2012) Rapid detection of rpoB gene mutations conferring rifampin resistance in Mycobacterium tuberculosis. Journal of Clinical Microbiology 50, 2433-2440.

Barroso, E.C., Mota, R.M.S., Santos, R.O., Sousa, A.L.O., Barroso, J.B., Rodrigues, J.L.N. (2003) Risk factors for acquired multidrug-resistant tuberculosis. Jornal de Pneumologia 29, 89-97.

Boehme, C.C., Nicol, M.P., Nabeta, P., Michael, J.S., Gotuzzo, E., Tahirli, R., Gler, M.T., Blakemore, R., Worodria, W., Gray, C., Huang, L., Caceres, T., Mehdiyev, R., Raymond, L., Whitelaw, A., Sagadevan, K., Alexander, H., Albert, H., Cobelens, F., Cox, H. Alland, D. \& Perkins, M.D. (2011) Feasibility, diagnostic accuracy, and effectiveness of decentralised use of the Xpert MTB/RIF test for diagnosis of tuberculosis and multidrug resistance: a multicentre implementation study. Lancet 377(9776), 1495-1505.

Chiang, C.Y., Slama, K. \& Enarson, D.A. (2007) Associations between tobacco and tuberculosis. International Journal of Tuberculosis and Lung Diseases 11, 258-262.

Chonde, T.M., Basra, D., Mfinanga, S.G.M., Range, N., Lwilla, F., Shirima, R.P., van Deun, A., Zignol, M., Cobelens, F.G., Egwaga, S. \& van Leth, F. (2010) National anti-tuberculous drug resistance study in Tanzania. International Journal of Tuberculosis and Lung Diseases 14, 967972.

Churchyard, G.J., Corbett, E.L., Kleinschmidt, I., Mulder, D. \& De Cock, K.M. (2000) Drug-resistant tuberculosis in South African gold miners: incidence and associated factors. International Journal of Tuberculosis and Lung Diseases 4, 433-440.

El Mahalli, A. \& Al-Qahtani, M.F. (2015) Predictors of drug resistance in tuberculosis patients in the Eastern Province, Saudi Arabia. Journal of the Egyptian Public Health Association 90, 24-28.

Escombe, A.R., Moore, D.A.J., Gilman, R.H., Pan, W., Navincopa, M., Ticona, E., Martinez, C., Caviedes, L., Sheen, P., Gonzalez, A., Noakes, C.J., Friedland, J.S. \& Vans, C.A. (2008) The infectiousness of tuberculosis patients coinfected with HIV. PLoS Medicine 5(9), 1387-1396.

Dean, A.S., Zignol, M., Falzon, D., Getahun, H. \& Floyd. K. (2014) HIV and multidrug-resistant tuberculosis: overlapping epidemics: An Individual Participant meta-analysis. European Respiratory Journal, 44(1), pp.247-251.

Liang, L., Wu, Q., Gao, L., Hao, Y., Liu, C., Xie, Y., Sun, H., Yan, X., Li, F., Li, H., Fang, H., Ning, N., Cui, Y. \& Han, L. (2012) Factors contributing to the high prevalence of multidrug-resistant tuberculosis : a study from China. Thorax 67, 632-638.

Marahatta, S.B., Kaewkungwal, J., Ramasoota, P. \& Singhasivanon, P. (2010) Risk factors of multidrug resistant tuberculosis in central Nepal: a pilot study. Kathmandu University Medical Journal 8, 392-397.

Matee, M., Mtei, L., Lounasvaara, T., Wieland-Alter, W., Waddell, R., Lyimo, J., Bakari, M., Pallangyo, K. \& von Reyn, C.F. (2008) Sputum microscopy for the diagnosis of HIVassociated pulmonary tuberculosis in Tanzania. BMC Public Health 8(1): 68.

Nagu, T.J., Aboud, S., Mwiru, R., Matee, M., Fawzi, W. \& Mugusi, F. (2015) Multi drug and other forms of drug resistant tuberculosis are uncommon among treatment naïve tuberculosis patients in Tanzania. PLoS ONE 10(4): 1-10.

NTLP (2013) Manual for the Management of Tuberculosis and Leprosy, Sixth Edition. National Tuberculosis and Leprosy Programme. Ministry of Health and Social Welfare, Dar es Salaam, United Republic of Tanzania.

Steingart, K.R., Schiller, I., Horne, D.J., Pai, M., Boehme, C.C. \& Dendukuri, N. (2014) Xpert ${ }^{\circledR M T B ~ / ~}$ RIF assay for pulmonary tuberculosis and rifampicin resistance in adults. The Cochrane Database Systematic Review (1):1-132.

WHO (2014) Companion handbook to the WHO Guidelines for the Programmatic Management of Drug-Resistant Tuberculosis. World Health Organization.

WHO (2006) The Stop Tb Strategy: Building on and enhancing DOTS to meet the TB-related Millennium Development Goals.

WHO (2013) Updated WHO Xpert expert Group Meeting Report, Geneva, Switzerland:World Health 


\section{Organization}

WHO (2015) WHO Global Tuberculosis Report 2015, 20 Avenue Appia, 1211 Geneva 27, Switzerland. Zignol, M., Hosseini, M.S., Wright, A., Weezenbeek, C.L., Nunn, P., Watt, C.J., Williams, B.G., Dye, C. (2006) Global incidence of multidrug-resistant tuberculosis. Journal of Infectious Disease $194,479-485$ 